\title{
Polynomially convex hulls of families of arcs
}

\author{
by \\ ZBigniew SŁodkowski (Chicago, IL)
}

\begin{abstract}
The paper is devoted to the study of polynomially convex hulls of compact subsets of $\mathbb{C}^{2}$, fibered over the boundary of the unit disc, such that all fibers are simple arcs in the plane and their endpoints form boundaries of two closed, not intersecting analytic discs. The principal question concerned is under what additional condition such a hull is a bordered topological hypersurface and, in particular, is foliated by a unique holomorphic motion. One of the main results asserts that this happens when the family of arcs satisfies the Continuous Cone Condition.
\end{abstract}

0. Motivation. The subject of this paper has originated from the following result of the author. (Throughout the paper, $D$ will denote the open unit disc in the complex plane $\mathbb{C}$, centered at $0 ; D(a, R)$ will stand for the disc of radius $R$, centered at $a$.)

TheOrem 0.1 ([Sł 1]). Let $X \subset \partial D \times \mathbb{C}$ be a compact set with all fibers $X_{\zeta}=\{w \in \mathbb{C}:(\zeta, w) \in X\}, \zeta \in \partial D$, nonempty, connected and simply connected. Let $Y:=\widehat{X}$ (the polynomial hull of $X$ ) and $S:=$ the topological boundary of $Y \backslash X$ relative to $D \times \mathbb{C}$. Then either $Y=X$ or $Y \backslash X$ can be represented as union of a family of analytic discs (= graphs of bounded analytic functions $f: D \rightarrow \mathbb{C}$ ). Furthermore $S$ has a unique foliation by such analytic discs.

The problems considered in this paper center around the following question. Assuming all $X_{\zeta}$ 's are simple arcs, when is $Y=\widehat{X}$ a topological hypersurface? In case $X$ is a $C^{2}$-regular surface this is a consequence of Forstneric [F]. On the other hand, Example 5.1 below shows that the hull of a family of arcs may have a nonempty interior. In view of these observations the task we undertake in this paper is that of finding minimal regularity assumptions for the hull $Y$ to be a topological hypersurface.

Apart from the intrinsic interest of this question for the study of polynomial hulls, our motivation comes also from the possibility of applications to constructions of holomorphic motions as suggested by [Sł 3]. The method

2000 Mathematics Subject Classification: Primary 32E20; Secondary 32A37. 
used by the author in [Sł 3] to give the proof of the "extended lambdalemma", alternative to that of [Sł 2], was based on the following construction. For some $R>0$, the disc $\bar{D}(0, R)$ in each fiber $\{\zeta\} \times \mathbb{C}$ was covered by a family of arcs $X_{\zeta}^{a}$ (which formed a foliation apart from $(\zeta, 0)$ ) and the hull of $\bigcup_{\zeta \in b D}\{\zeta\} \times X_{\zeta}^{a}$ has been shown to admit, for each $a$, a unique holomorphic motion tracing it. The union of all these partial holomorphic motions for different $a$ 's yielded a holomorphic motion of $\mathbb{C}$ over $D$. The connection with the problems of this paper comes from the observation that if the hull $Y^{a}=\widehat{X^{a}}$ is a topological hypersurface, then it must have a unique foliation by analytic discs, i.e. it is traced by a unique holomorphic motion. (Cf. Corollary 2.1 below.) If the same conclusion is obtained under low enough regularity assumptions on $X^{a}$, the outlined method of construction of holomorphic motions will become sufficiently general and flexible.

In the present paper we concentrate on the study of hulls, postponing applications to holomorphic motions for another occasion.

1. Outline of results. We will denote by $A(D)$ the algebra of all functions holomorphic on the unit disc $D$ and continuously extensible to $\bar{D}$.

With the exception of Section 2, we will consider families of arcs of the following kind.

Basic Situation 1.1. $X$ is a compact subset of $b D \times \mathbb{C}$ such that

(i) there exist two functions $a, b \in A(D)$ satisfying $a(z) \neq b(z)$ for all $z \in \bar{D}$;

(ii) for every $\zeta \in b D$, the fiber $X_{\zeta}$ is a simple topological arc joining $a(\zeta)$ and $b(\zeta)$.

We will denote by $Y$ the polynomially convex hull of $X$.

To this basic set-up we will be adding more regularity assumptions on $\operatorname{arcs} X_{\zeta}$ depending on the results in question.

In Section 3 we assume that all $X_{\zeta}$ 's are $K$-quasiarcs with a uniform constant $K$. While we will show later that $Y$ does not have to be a hypersurface (Example 5.1), we prove (Theorem 3.1) that the boundary of $Y=\widehat{X}$ in $\mathbb{C}^{2}$ is a union of two topological hypersurfaces, each bordered by (the union of) $X$ and the end-discs $a, b$, and whose fibers are quasiarcs with a uniform constant. As a corollary, such a hull is a hypersurface if and only if it has empty interior in $\mathbb{C}^{2}$.

A technical condition on the position of the end-discs $a, b$ (cf. Definition, Section 3) has to be assumed in this result. The role of this condition is to ensure that the end-discs $a, b$ are contained in the boundary of the hull $Y$. We show in Section 5 that if this condition is omitted, then the part over $D$ of the end-disc $a$ or $b$ might be contained in the interior of $Y$ (cf. Example 5.2). 
In Section 4 we assume that $\operatorname{arcs} X_{\zeta}, \zeta \in b D$, satisfy the following (one-sided) Continuous Cone Condition.

Continuous Cone Condition 1.2. There is a function $x(\zeta, w)=$ $\exp (i \psi(\zeta, w))$, for $\zeta \in b D, w \in X_{\zeta}$, with $\psi: X \rightarrow \mathbb{R}$ continuous, and positive numbers $\alpha, \beta>0$, such that the cones

$$
C(\zeta, w)=\left\{v=w+r e^{i \theta} x(\zeta, w):|\theta| \leq \alpha, 0 \leq r \leq \beta\right\}
$$

satisfy

$$
\begin{gathered}
C(\zeta, w) \cap X_{\zeta}=\{w\}, \quad(\zeta, w) \in X, \\
C(\zeta, a(\zeta)) \cap C(\zeta, b(\zeta))=\emptyset, \quad \zeta \in b D .
\end{gathered}
$$

Under this assumption the hull of $X$ turns out to be a topological hypersurface (Theorem 4.1). Its fibers must be quasiarcs by the already mentioned Theorem 3.1, but one can prove, in fact, that they also satisfy the Continuous Cone Condition.

In the proof, a crucial role is played by functions with vanishing mean oscillation, or, more precisely, by so-called quasicontinuous functions (i.e. the class $L^{\infty} \cap \mathrm{VMO}$ ). We prove in Section 2 (Proposition 2.3) that the boundary values of analytic discs in the hull of $X$ such that every $X_{\zeta}$, $\zeta \in b D$, is nowhere dense in $\mathbb{C}$ are quasicontinuous functions. This enables us to apply the techniques of VMO in the context of Section 4.

Once we know that the hull $Y$ is a hypersurface and so is traced by a unique holomorphic motion over $D$, it is natural to ask when this motion can be extended to a continuous isotopy over $\bar{D}$. We show (cf. Example 5.3) that even if $X$ is a continuous family of uniformly $C^{1}$-regular arcs (in fact real-analytic), its hull $Y$ may contain two analytic discs which, while disjoint (by necessity) over $D$ and extending continuously over $\bar{D}$, have nevertheless nonempty intersection over $b D$. We postpone investigation of sufficient conditions to another paper.

2. Some properties of hulls with connected fibers. In this section we collect several auxiliary facts about hulls of sets $X$ whose boundary fibers $X_{\zeta}$ are connected and simply connected, but not necessarily arcs.

Corollary 2.1. Let $Y=\widehat{X}$ be as in Theorem 0.1. Assume that it has a nowhere dense fiber $Y_{a}$ for some $a \in D$. Then no two distinct analytic discs contained in $Y$ can intersect over $D$. If there are two analytic discs in $Y$ intersecting over $D$, then $Y$ has nonempty interior in $\mathbb{C}^{2}$.

Proof. By Theorem 0.1, $S$ can be foliated by analytic discs; choose one such foliation and let

$$
(z, w) \mapsto f_{z}(w): D \times S_{a} \rightarrow \mathbb{C}
$$


be the corresponding holomorphic motion. In particular

$$
f_{z}\left(S_{a}\right)=S_{z}, \quad z \in D ; \quad f_{a}=\operatorname{id}_{S_{a}} .
$$

(In fact, our proof shows that the foliation of $S$ is unique.) Define $U_{a}=$ $\mathbb{C} \backslash S_{a}$. By the "extended lambda lemma" (cf. [Sł 2, Theorem 1.3]), there is a holomorphic motion $F_{z}$ of $U_{a}$ disjoint from $S$, i.e.

$$
(z, w) \mapsto F_{z}(w): D \times U_{a} \rightarrow \mathbb{C}, \quad F_{z}\left(S_{a}\right) \cap S_{z}=\emptyset, \quad z \in D .
$$

Let $g \in A(D)$ be any analytic disc in $Y$. Set $b:=g(a)$. We now define two holomorphic motions of $U_{a} \cup\{b\}$,

$$
(z, w) \mapsto F_{z}^{j}(w): D \times\left(U_{a} \cup\{b\}\right) \rightarrow \mathbb{C}, \quad j=1,2,
$$

by letting $F_{z}^{j} \mid U_{a}=F_{z}, j=1,2, F_{z}^{1}(b):=g(z), F_{z}^{2}(b):=f_{z}(b), z \in D$. Since $F^{1}, F^{2}$ are two holomorphic motions of $U_{a} \cup\{b\}$ which are identical on the dense subset $U_{a}$, the standard lambda lemma [MSS] implies that they are equal, in particular $g(z)=f_{z}(b)$, which implies the uniqueness of the disc $g$. Consequently, distinct discs have to be disjoint.

Thus, if there are two intersecting analytic discs, at least one fiber, say $Y_{a}, a \in D$, contains a nonempty open set, say $V$. Then the set $\left\{\left(z, F_{z}(w)\right)\right.$ : $z \in D, w \in V\}$ is a nonempty open subset of $Y$.

We will denote clusters as follows:

$$
\mathrm{Cl}(g, \zeta)=\left\{w \in \mathbb{C}: \exists\left(z_{n}\right) \subset D, \lim z_{n}=\zeta, \lim g\left(z_{n}\right)=w\right\} .
$$

Proposition 2.2. Let $X^{j}, j=1,2$, be compact subsets of $b D \times \mathbb{C}$ with connected and simply connected fibers. Assume that $X^{1}, X^{2}$ are disjoint, and furthermore, there are two functions $g_{1}, g_{2} \in H^{\infty}(D)$ such that

$$
\mathrm{Cl}\left(g_{j}, \zeta\right) \subset X_{\zeta}^{j}, j=1,2, \quad \zeta \in b D, \quad g_{1}(z) \neq g_{2}(z), \quad z \in D .
$$

Then the polynomial hulls $Y^{1}=\widehat{X^{1}}$ and $Y^{2}=\widehat{X^{2}}$ are disjoint.

Proof. Seeing that $z \mapsto Y^{j}(z): \bar{D} \rightarrow 2^{\mathbb{C}}, j=1,2$, are upper semicontinuous set-valued functions such that $Y^{1}(\zeta) \cap Y^{2}(\zeta)=\emptyset$ for $|\zeta|=1$ (because $Y^{j}(\zeta)=X^{j}(\zeta)$ for $|\zeta|=1$ ), we conclude that there is $0<r_{0}<1$ such that

$$
Y^{1}(r \zeta) \cap Y^{2}(r \zeta)=\emptyset, \quad r \in\left[r_{0}, 1\right),|\zeta|=1 .
$$

For each $j=1,2$, the function $g_{j}$ constitutes a holomorphic motion of one point $g_{j}(0)$. On the other hand, as in the last proof, there is also a holomorphic motion of $U_{0}^{j}$, tracing the sets $U_{z}^{j}:=\mathbb{C} \backslash Y_{z}^{j}, z \in D$ (cf. [Sł 2]). Altogether, they give a holomorphic motion of the set $U_{0}^{j} \cup\left\{g_{j}(0)\right\}$, which, by [Sł 2], can be extended to a holomorphic motion of $\mathbb{C}$. Restricting it to $Y_{0}^{j}$, we obtain a motion with the following properties:

$$
\begin{gathered}
(z, w) \mapsto f_{z}^{j}(w): D \times Y_{0}^{j} \rightarrow \mathbb{C}, \\
f_{z}^{j}\left(Y_{0}^{j}\right)=Y_{z}^{j}, \quad f_{z}^{j}\left(g_{j}(0)\right)=g_{j}(z), \quad z \in D .
\end{gathered}
$$


By (2.2), for every $\left(w^{1}, w^{2}\right) \in Y_{0}^{1} \times Y_{0}^{2}$ the function

$$
\zeta \mapsto f_{r \zeta}^{1}\left(w_{1}\right)-f_{r \zeta}^{2}\left(w_{2}\right): b D \rightarrow \mathbb{C} \backslash\{0\}
$$

does not vanish, and has a well defined winding number, which we denote by $n\left(w_{1}, w_{2}\right)$. By the uniform continuity of the motions $f^{j}$ on compact subsets of $D \times \mathbb{C}$, due to the lambda lemma (cf. [MSS]), the function $n: Y_{0}^{1} \times Y_{0}^{2} \rightarrow \mathbb{Z}$ is continuous, and so constant, as $Y_{0}^{1} \times Y_{0}^{2}$ is connected. On the other hand $n\left(g_{1}(0), g_{2}(0)\right)=\operatorname{ind}\left(g_{1}-g_{2}\right)=0$, by the assumption $(2.1)$.

Thus $f_{z}^{1}\left(w_{1}\right) \neq f_{z}^{2}\left(w_{2}\right)$ for $|z| \leq r$. As $r \rightarrow 1-0$, and since our holomorphic motions trace $Y^{1} \backslash X^{1}$ and $Y^{2} \backslash X^{2}$, we conclude that $Y^{1}$ and $Y^{2}$ are disjoint.

Proposition 2.3. Let $X$ be a compact subset of $b D \times \mathbb{C}$ with connected, simply connected and nowhere dense (in $\mathbb{C})$ fibers. Then for any analytic disc in $Y$ of the form $\operatorname{graph}(f), f \in H^{\infty}(D)$, its boundary values $f^{*}$ are quasicontinuous, i.e. $f^{*} \in Q C=L^{\infty} \cap \mathrm{VMO}$.

Proof. Since fibers of $X$ are simply connected and nowhere dense, we can apply a parametrized version of Mergelian's theorem (cf. [F, Lemma 7] or see [Sł 4]) for a more direct proof using decomposition of unity) to show that for every $\varepsilon>0$ there is a polynomial in $z$

$$
p_{N}\left(e^{i \theta}, z\right)=\sum_{j=0}^{N} k_{j}\left(e^{i \theta}\right) z^{j}
$$

such that $\left|\bar{z}-p_{N}\left(e^{i \theta}, z\right)\right|<\varepsilon$ for $\left(e^{i \theta}, z\right) \in X$, where $k_{0}, \ldots, k_{N}: b D \rightarrow \mathbb{C}$ are continuous functions. Set $F_{N}\left(e^{i \theta}\right):=\sum_{j=0}^{N} k_{j}\left(e^{i \theta}\right) f\left(e^{i \theta}\right)^{j}$, defined a.e. on $b D$. Denote by $C$ the algebra of continuous functions on $b D$. Since $H^{\infty}+C$ is a closed subalgebra of $L^{\infty}$ (cf. [G, Theorem IX.2.2]), it contains $f_{N}, N=$ $1,2, \ldots$, as well as their uniform limit $\bar{f}$, since $\left\|\bar{f}-f_{N}\right\|<\varepsilon$. We conclude that $f \in\left(\overline{H^{\infty}}+C\right) \cap\left(H^{\infty}+C\right)$, which is equal to $Q C$ by [G, Theorem IX.2.3].

\section{Hulls with quasiarc boundary fibers}

Definition. Let $X, a, b$ be as in Basic Situation 1.1. We say that $X$ satisfies the Continuous Cone Condition along the disc a if there are positive numbers $\alpha, \beta$ and a continuous function $x: b D \rightarrow b D$ such that $\alpha \in(0, \pi / 2)$ and $C(\zeta) \cap X_{\zeta}=\{a(\zeta)\}$ for $\zeta \in b D$, where

$$
C(\zeta):=\left\{z=a(\zeta)+r e^{i \theta} x(\zeta): 0 \leq r \leq \beta, \theta \in[-\alpha, \alpha]\right\} .
$$

Theorem 3.1. Let $X, a, b$ be as in Basic Situation 1.1. Assume that $X$ satisfies the Continuous Cone Condition along the discs $a, b$ and that all fibers $X_{\zeta}, \zeta \in b D$, are quasiarcs with a uniform constant $K$. Then the 
boundary $\left(\right.$ in $\left.\mathbb{C}^{2}\right)$ of the polynomial hull $Y$ of $X$ is the union of two topological hypersurfaces $\Sigma^{1}, \Sigma^{2}$, whose fibers (over $z \in \bar{D}$ ) are quasiarcs with a uniform constant, and with $X \cup \operatorname{graph}(a) \cup \operatorname{graph}(b)$ as their common boundary. Thus, if $Y$ has empty interior, then it is a topological hypersurface $Y=\Sigma^{1}=\Sigma^{2}$.

Proof. Let $C^{a}(\zeta), C^{b}(\zeta)$ denote continuous families of (truncated) cones as in the above definition, that is,

$$
\begin{aligned}
& C^{a}(\zeta):=\left\{z=a(\zeta)+r e^{i \theta} x(\zeta): 0 \leq r \leq \beta, \theta \in[-\alpha, \alpha]\right\}, \\
& C^{b}(\zeta):=\left\{z=b(\zeta)+r e^{i \theta} y(\zeta): 0 \leq r \leq \beta, \theta \in[-\alpha, \alpha]\right\} .
\end{aligned}
$$

The conditions

$$
C^{a}(\zeta) \cap X_{\zeta}=\{a(\zeta)\}, \quad C^{b}(\zeta) \cap X_{\zeta}=\{b(\zeta)\}, \quad \text { for } \zeta \in b D,
$$

and the assumed properties of $X$ imply that $x, y$ (which do not vanish) are of index zero. We can also assume without loss of generality (by diminishing $\alpha$ and $\beta$ if necessary) that $x$ and $y$ are $C^{\infty}$ smooth. Then the functions

$$
\begin{aligned}
& \widetilde{x}(\zeta):=\exp [-T(\arg x(\zeta))+i \arg x(\zeta)], \\
& \widetilde{y}(\zeta):=\exp [-T(\arg y(\zeta))+i \arg y(\zeta)]
\end{aligned}
$$

(where the branches of "arg" are chosen continuous, hence smooth, and $T$ of a function denotes its conjugate function with mean zero, i.e. $T$ is the Hilbert operator) are smooth and have smooth holomorphic extensions to $\bar{D}$. Consequently, the functions

$$
a+\frac{r}{2\|\widetilde{x}\|_{\infty}} \widetilde{x}, \quad b+\frac{r}{2\|\widetilde{y}\|_{\infty}} \widetilde{y}
$$

belong to $A(D)$. Furthermore, they are continuous selections, over $b D$, of the set-valued functions $C^{a}(\zeta), C^{b}(\zeta)$, respectively. If we now choose functions $g_{0}, h_{0} \in C^{\infty} \cap A(D)$ so that they approximate the functions (3.2) closely enough in the uniform norm, then there are real numbers $\delta, \eta$ such that

$$
\begin{gathered}
g_{0}(\zeta) \in \operatorname{Int} C^{a}(\zeta), \quad h_{0}(\zeta) \in \operatorname{Int} C^{b}(\zeta), \\
0<s:=\min \left\{\left|g_{0}(\zeta)-a(\zeta)\right|,\left|h_{0}(\zeta)-b(\zeta)\right|: \zeta \in b D\right\}, \\
C_{2 \eta}^{a}(\zeta) \subset C^{a}(\zeta), \quad C_{2 \eta}^{b}(\zeta) \subset C^{b}(\zeta), \quad \zeta \in b D,
\end{gathered}
$$

where

$$
\begin{aligned}
& C_{2 \eta}^{a}(z):=\left\{w=a(z)+r e^{i \theta}\left(g_{0}(z)-a(z)\right): 0 \leq r \leq 1,|\theta| \leq 2 \eta\right\}, \\
& C_{2 \eta}^{b}(z):=\left\{w=b(z)+r e^{i \theta}\left(h_{0}(z)-b(z)\right): 0 \leq r \leq 1,|\theta| \leq 2 \eta\right\} .
\end{aligned}
$$

Observe that for $0<r \leq 1$ and $|\theta| \leq 2 \eta$, the functions $z \mapsto w: \bar{D} \rightarrow \mathbb{C}$ defined by $w=a(z)+r e^{\overline{i \theta}}\left(g_{0}(z)-a(z)\right.$ ) (which cover $C_{2 \eta}^{a}(z) \backslash\{a(z)\}$ have graphs (over $\bar{D}$ ) disjoint from that of $a$, and boundary values disjoint from $X$, 
and so by Proposition 2.2 their graphs are disjoint from $Y=\widehat{X}$. Thus

$$
C_{2 \eta}^{a}(z) \cap Y_{z}=\{a(z)\}, \quad C_{2 \eta}^{b}(z) \cap Y_{z}=\{b(z)\} .
$$

By (3.1), (3.5) and Janiszewski's Theorem, for $r<\min _{z \in \bar{D}}|a(z)-b(z)|$ the following sets are connected and simply connected:

$$
\begin{gathered}
C_{2 \eta}^{a}(z) \cup Y_{z} \cup C_{2 \eta}^{b}(z), \quad z \in \bar{D} \\
\left\{(z, w) \in \bar{D} \times \mathbb{C}: w \in C_{2 \eta}^{a}(z) \cup Y_{z} \cup C_{2 \eta}^{b}(z)\right\} .
\end{gathered}
$$

Furthermore the functions

$$
g_{0}^{ \pm 2 \eta}=a+\frac{1}{2} e^{ \pm 2 i \eta}\left(g_{0}-a\right), \quad h_{0}^{ \pm 2 \eta}=b+\frac{1}{2} e^{ \pm 2 i \eta}\left(h_{0}-a\right)
$$

have graphs contained in the boundary of the set (3.6). Define further

$$
p^{1}:=\frac{a+b}{2}+i \chi \frac{a-b}{2}, \quad p^{2}:=\frac{a+b}{2}-i \chi \frac{a-b}{2},
$$

where $\chi$ is a positive constant large enough so that $p^{1}(z), p^{2}(z)$ do not belong to (3.6) for any $z \in \bar{D}$.

The following assertion is now topologically obvious.

Assertion 1. There exist two families of simple smooth arcs $\gamma_{\zeta}^{1}, \gamma_{\zeta}^{2}$, for $\zeta \in b D$, such that

(i) $\gamma_{\zeta}^{1}$ has endpoints $g_{0}^{2 \eta}(\zeta), h_{0}^{-2 \eta}(\zeta)$ and $\gamma_{\zeta}^{2}$ has endpoints $h_{0}^{2 \eta}(\zeta)$, $g_{0}^{-2 \eta}(\zeta)$, with the orientation indicated by the order of endpoints, while

$$
\gamma_{\zeta}^{1} \cup \gamma_{\zeta}^{2} \subset \mathbb{C} \backslash\left[C_{2 \eta}^{a}(\zeta) \cup X_{\zeta} \cup C_{2 \eta}^{b}(\zeta)\right], \quad \zeta \in b D
$$

(ii) $\bigcup_{\zeta \in b D}\{\zeta\} \times \gamma_{\zeta}^{1}$ and $\bigcup_{\zeta \in b D}\{\zeta\} \times \gamma_{\zeta}^{2}$ are $C^{2}$-smooth surfaces;

(iii) for every $\zeta \in b D$, the point $p^{1}$ (resp. $p^{2}$ ) belongs to the bounded connected component of the complement of $\gamma_{\zeta}^{1} \cup C_{2 \eta}^{a}(\zeta) \cup X_{\zeta} \cup C_{2 \eta}^{b}(\zeta)$ (resp. $\gamma_{\zeta}^{2} \cup C_{2 \eta}^{a}(\zeta) \cup X_{\zeta} \cup C_{2 \eta}^{b}(\zeta)$ ) and these components are disjoint.

The arcs $\gamma$ can be constructed by standard ODE techniques similarly to [Sł 2, Section 2]. We omit further details.

It is easy to see that the arcs $\gamma$ have uniformly bounded quasiarc constants. Consider yet the following circular and straight arcs:

$$
\begin{aligned}
\sigma_{a}^{ \pm}(\zeta) & :=\left\{a(\zeta)+(1 / 2) e^{ \pm i \theta}\left(g_{0}-a\right)(\zeta): \eta \leq \theta \leq 2 \eta\right\} \\
\tau_{a}^{ \pm}(\zeta) & :=\left\{a(\zeta)+s e^{ \pm i \eta}\left(g_{0}-a\right)(\zeta): 0 \leq s \leq 1\right\},
\end{aligned}
$$

as well as $\sigma_{b}^{ \pm}(\zeta), \tau_{b}^{ \pm}(\zeta)$, defined analogously.

Assertion 2. The paths

$$
\begin{aligned}
& X_{\zeta}^{1}:=X_{\zeta} \cup \tau_{a}^{+}(\zeta) \cup \sigma_{a}^{+}(\zeta) \cup \gamma_{\zeta}^{1} \cup \sigma_{a}^{-}(\zeta) \cup \tau_{a}^{-}(\zeta), \\
& X_{\zeta}^{2}:=X_{\zeta} \cup \tau_{b}^{+}(\zeta) \cup \sigma_{b}^{+}(\zeta) \cup \gamma_{\zeta}^{2} \cup \sigma_{b}^{-}(\zeta) \cup \tau_{b}^{-}(\zeta)
\end{aligned}
$$


are Jordan curves and quasicircles with a uniform constant, for $\zeta \in b D$. Furthermore

$$
X_{\zeta}^{1} \cap X_{\zeta}^{2}=X_{\zeta}, \quad \zeta \in b D .
$$

In addition, the bounded connected components of $\mathbb{C} \backslash X_{\zeta}^{1}$ and $\mathbb{C} \backslash X_{\zeta}^{2}$ are disjoint and contain $p^{1}$ and $p^{2}$ respectively.

We omit the details. (They are elementary but somewhat delicate since a Jordan curve which is the union of several quasiarcs does not have to be a quasicircle in general.)

Now we let

$$
\begin{array}{rlrl}
X^{1} & :=\bigcup_{\zeta \in b D}\{\zeta\} \times X_{\zeta}^{1}, & X^{2}:=\bigcup_{\zeta \in b D}\{\zeta\} \times X_{\zeta}^{2}, \\
Y^{1}:=\widehat{X^{1}}, & Y^{2}:=\widehat{X^{2}} .
\end{array}
$$

We will first prove that $Y=Y^{1} \cap Y^{2}$ and then deduce from this the required properties of $Y$. Denote by $U^{j}, j=1,2$, the interior of $Y^{j}$ relative to $\bar{D} \times \mathbb{C}$. By Assertion 2 the set $U^{j}$ contains a constant analytic section $p^{j}$, which by [Sł 1, Lemma 4.6] implies that

$$
\left(U^{j}\right)_{\zeta}=\operatorname{Int} Y_{\zeta}^{j}=Y_{\zeta}^{j} \backslash X_{\zeta}^{j}, \quad j=1,2, \zeta \in b D .
$$

This means that $\overline{S^{j}} \backslash S^{j}=X^{j}, j=1,2$, where $S^{j}=$ the boundary of $Y^{j}$ relative to $D \times \mathbb{C}$, i.e. $S^{j}=\bigcup_{z \in D}\{z\} \times b Y_{z}^{j}$.

ASSERTION 3. $S^{j}, j=1,2$, has a unique foliation by analytic discs. Furthermore, all fibers of $\overline{S^{j}}=S^{j} \cup X^{j}, j=1,2$, are quasicircles with a uniform constant, hence Jordan curves.

With the exception of the statement that $\overline{S^{j}}$ is a bordered hypersurface, all the properties follow from [Sł 1, Theorems 1.1 and 7.1]. To conclude that $\overline{S^{j}}$ is a bordered hypersurface, one has to review the proof of Theorem 7.2 in [Sł 1] and to realize that the Continuous Cone Condition was used there only to ensure that (in the present notation) $\overline{S^{j}} \backslash S^{j} \subset X^{j}$, which in our case is ensured by the specific construction (in particular Int $Y_{z}^{j}$ for $z \in \bar{D}$ admits a constant selection $p_{j}$ ).

Consider $S^{1}$ and the following four pairwise disjoint continuous analytic discs contained in $S^{1}$ :

$$
a, \quad g_{0}^{\eta}=a+(1 / 2) e^{i \eta}\left(g_{0}-a\right), \quad h_{0}^{-\eta}=b+(1 / 2) e^{-i \eta}\left(h_{0}-b\right), \quad b .
$$

It is now clear, by Assertion 3, that the complement of these four discs in $S^{1}$ is a union of four connected components, pairwise disjoint, which we can 
label $S_{1}^{1}, S_{2}^{1}, S_{3}^{1}, S_{4}^{1}$, such that

$$
\begin{aligned}
& \overline{S_{1}^{1}} \backslash S_{1}^{1}=\operatorname{gr}(a)+\operatorname{gr}\left(g_{0}^{\eta}\right), \quad \overline{S_{2}^{1}} \backslash S_{2}^{1}=\operatorname{gr}\left(g_{0}^{\eta}\right)+\operatorname{gr}\left(h_{0}^{-\eta}\right), \\
& \overline{S_{3}^{1}} \backslash S_{3}^{1}=\operatorname{gr}\left(h_{0}^{-\eta}\right)+\operatorname{gr}(b), \quad \overline{S_{4}^{1}} \backslash S_{4}^{1}=\operatorname{gr}(b)+\operatorname{gr}(a),
\end{aligned}
$$

(where "gr" stands for graph) and their fibers over $b D$ are as follows:

$$
\begin{array}{ll}
\left(\overline{S_{1}^{1}}\right)_{\zeta}=\tau_{a}^{+}(\zeta), & \left(\overline{S_{2}^{1}}\right)_{\zeta}=\sigma_{a}^{+}(\zeta) \cup \gamma_{\zeta}^{1} \cup \sigma_{b}^{-}(\zeta), \\
\left(\overline{S_{3}^{1}}\right)_{\zeta}=\tau_{b}^{-}(\zeta), & \left(\overline{S_{4}^{1}}\right)_{\zeta}=X_{\zeta} .
\end{array}
$$

Assertion 4. $S_{1}^{1} \cup \overline{S_{2}^{1}} \cup S_{3}^{1}$ is disjoint from $Y^{2}$.

Since $\overline{S_{2}^{1}}$ is foliated by analytic discs, it is contained in the polynomial hull of the compact set

$$
\bigcup_{|\zeta|=1}\{\zeta\} \times\left(\sigma_{a}^{+}(\zeta) \cup \gamma_{\zeta}^{1} \cup \sigma_{b}^{-}(\zeta)\right)
$$

which is disjoint from

$$
Y^{2} \mid b D=\bigcup_{|\zeta|=1}\{\zeta\} \times \widehat{X_{\zeta}^{2}},
$$

because, by Assertion $2, X_{\zeta}^{1} \cap X_{\zeta}^{2}=X_{\zeta}$ and $\left(\widehat{X_{\zeta}^{1}} \backslash X_{\zeta}^{1}\right) \cap\left(\widehat{X_{\zeta}^{2}} \backslash X_{\zeta}^{2}\right)=\emptyset$ for $\zeta \in b D$. Note that $g_{0}^{\eta}$ and $a$ are two disjoint continuous analytic discs with boundaries in the sets (3.11) and (3.12) respectively, and so by Proposition 2.2 the hulls of the two sets are disjoint, in particular $\overline{S_{2}^{1}}$ and $Y^{2}$ are disjoint. As for $\overline{S_{1}^{1}}$, since its boundary fibers $\left(\overline{S_{1}^{1}}\right)_{\zeta}$ are straight line segments joining the two disjoint continuous analytic discs $a$ and $g_{0}^{\eta}$, by the classical linear Riemann-Hilbert problem every analytic disc with boundary values in these segments must be of the form $t a+(1-t) g_{0}^{\eta}, 0<t<1$. For $t \in(0,1]$, such a disc is disjoint from the graph of $a$, and by the properties of the cones $C_{\zeta}^{a}$, its boundary is disjoint from the fibers $Y_{\zeta}^{2},|\zeta|=1$, and so, by Proposition 2.2, the discs $t a+(1-t) g_{0}^{\eta}, 0<t \leq 1$, are disjoint from the hull $Y^{2}$. Since every point of $S_{1}^{1}$ belongs to one of these discs, $S_{1}^{1} \cap Y^{2}=\emptyset$. The argument that $S_{3}^{1} \cap Y^{2}=\emptyset$ is identical. The proof of Assertion 4 is complete. On the other hand, as $\left(\overline{S_{4}^{1}}\right)_{\zeta}=X_{\zeta}$, we get $\overline{S_{4}^{1}} \subset Y=\widehat{X}$. Since

$$
b Y_{z}^{1}=S_{z}^{1}=\left(S_{1}^{1} \cup \overline{S_{2}^{1}} \cup S_{3}^{1}\right)_{z} \cup\left(\overline{S_{4}^{1}}\right)_{z}, \quad z \in D,
$$

we infer, in view of Assertion 4, that $b Y_{z}^{1} \cap Y_{z}^{2} \subset Y_{z}, z \in D$, and by a symmetrical argument, $b Y_{z}^{2} \cap Y_{z}^{1} \subset Y_{z}, z \in D$. Put $K=Y_{z}$ and $L=Y_{z}^{1} \cap Y_{z}^{2}$. By the above remarks, $b L=\left(b Y_{z}^{1} \cap Y_{z}^{2}\right) \cup\left(b Y_{z}^{2} \cap Y_{z}^{1}\right) \subset b K$. Furthermore, $K$ is connected and simply connected, $K \subset L$ and $L$ is simply connected as the intersection of two simply connected compact sets $Y_{z}^{1}$ and $Y_{z}^{2}$. 
EXERCISE. Let $K$ and $L$ be two planar compact sets such that $K \subset L$ and $b L \subset b K$. Then $K=L$.

This proves the desired relation $Y=Y^{1} \cap Y^{2}$.

Let now $\Sigma^{1}=\overline{S_{4}^{1}}$, and let $\Sigma^{2}$ be the object constructed in the identical way for $Y^{2}$. By Assertion 3, and the discussion following it, $\Sigma^{1}, \Sigma^{2}$ are two bordered topological hypersurfaces, homeomorphic to $\bar{D} \times[0,1]$, with common boundary surface $\operatorname{gr}(a) \cup \operatorname{gr}(b) \cup X$, and with all fibers $\Sigma_{z}^{1}, \Sigma_{z}^{2}$, $z \in \bar{D}$, quasicircles with a uniform constant.

As observed in the proof of Corollary 2.1, if the hull $Y$ has empty interior in $\mathbb{C}^{2}$, then all fibers $Y_{z}, z \in D$, have empty interior in the plane. But if the boundary of a nowhere dense planar compact $Y_{z}$ is the union of two Jordan $\operatorname{arcs} \Sigma_{z}^{1}$ and $\Sigma_{z}^{2}$ with common endpoints, these arcs have to be identical. Thus $\Sigma_{z}^{1}=\Sigma^{2}$.

4. Application of the Continuous Cone Condition. The following is the main result of this section.

Theorem 4.1. Let $X=\bigcup\left\{e^{i \theta}\right\} \times X_{e^{i \theta}}$ be a continuous family of arcs, as in Basic Situation 1.1. Assume the arcs satisfy the Continuous Cone Condition 1.2. Then the polynomial hull $Y=\widehat{X}$ is a (bordered) topological hypersurface all of whose fibers $Y_{z}, z \in \bar{D}$, are $K$-quasicircles (with a uniform $K$ ).

We will derive this theorem from the following lemma.

Lemma 4.2. Let $X$ satisfy the conditions of the above theorem. Let $L \subset$ $b D \times \mathbb{C}$ be a compact set with simply connected fibers containing $X$. Assume that the family of cones for $X$ given by the Continuous Cone Condition satisfies

$$
C(\zeta, w) \cap L_{\zeta}=\{w\}, \quad(\zeta, w) \in X .
$$

Then $Y=\widehat{X}$ is contained in the topological boundary of the hull $\widehat{L}$.

Proof of Theorem 4.1. Applying the lemma with $L=X$ we see that $Y=\widehat{X}$ is contained in its own boundary, i.e. is nowhere dense, and so by Theorem 3.1 the hull $Y$ is a topological hypersurface with quasiarc fibers. Note that Theorem 3.1 is applicable, because by the remarks in [Sł 1, proof of Lemma 6.4], arcs satisfying the Continuous Cone Condition are quasiarcs with a uniform constant.

The proof of the lemma will use in a crucial way the properties of the class of quasicontinuous functions, denoted $Q C$, which was introduced by Sarason as

$$
Q C:=\left(H^{\infty}+C\right) \cap\left(\overline{H^{\infty}}+C\right) .
$$


All these spaces are spaces of functions on the unit circle $b D=\{|z|=1\}$. In particular, $C$ denotes the space of all continuous complex-valued functions on $b D$. Below we list the properties of $Q C$ we are going to use. Some of them are slightly adapted; otherwise their proofs are in Garnett [G, Chapter IX].

Proposition 4.3. (a) $Q C$ is a closed (commutative) $C^{*}$-subalgebra of $L^{\infty}$.

(b) $Q C=L^{\infty} \cap V M O$.

(c) If $g \in Q C$ and $\psi(\zeta, w)$ is a continuous function with compact domain containing almost all points $(\zeta, g(\zeta)), \zeta \in b D$, then the function $\chi(\zeta):=\psi(\zeta, g(\zeta)), \zeta \in b D$, belongs to $Q C$.

(d) (Asymptotic multiplicativity of the Poisson integral) For $p, q \in Q C$ and $r:=p q \in Q C$, let $p(z), q(z), r(z)$ denote the values at $z$ of the Poisson integrals of $p, q, r$ respectively. Then

$$
\lim _{|z| \rightarrow 1-0}|p(z) q(z)-r(z)|=0 .
$$

(e) If $p, q \in Q C$ and $\psi: \bar{D}(0, R) \times \bar{D}(0, R) \rightarrow \mathbb{C}$ is a continuous function, where $R \geq\|p\|_{\infty},\|q\|_{\infty}$, put $\chi(\zeta):=\psi(p(\zeta), q(\zeta)), \zeta \in b D$, a.e. $|d \zeta|$, and let $\chi(z),|z|<1$, denote the value of the Poisson integral at $z$. Then

$$
\lim _{|z| \rightarrow 1-0}(\psi(p(z), q(z))-\chi(z))=0 .
$$

Proof of $(e)$. For $\varepsilon>0$, there is a polynomial in $w, \bar{w}, z, \bar{z}$, say $\psi_{\varepsilon}$, such that

$$
\left|\psi(w, v)-\psi_{\varepsilon}(w, v)\right| \leq \varepsilon, \quad(w, v) \in \bar{D}(0, R) \times \bar{D}(0, R) .
$$

Put $\chi_{\varepsilon}(\zeta):=\psi_{\varepsilon}(p(\zeta), q(\zeta))$, and let $\chi_{\varepsilon}(z),|z|<1$, denote the Poisson integral. Since Poisson integral is asymptotically multiplicative on the algebra $Q C$ (properties (d) and (a) above), and is obviously additive, we deduce by a finite number of applications that the asymptotic formula holds for polynomials:

$$
\lim _{|z| \rightarrow 1-0}\left(\psi_{\varepsilon}(p(z), q(z))-\chi_{\varepsilon}(z)\right)=0 .
$$

Applying this together with

$$
\left|\chi(w, v)-\chi_{\varepsilon}(w, v)\right| \leq \varepsilon, \quad(w, v) \in \bar{D}(0, R) \times \bar{D}(0, R),
$$

we conclude by the triangle inequality that

$$
\limsup _{|z| \rightarrow 1-0}|\psi(p(z), q(z))-\chi(z)| \leq 2 \varepsilon,
$$

for every $\varepsilon>0$.

The next proposition restates classical results (cf. [G, Lemma IV.3.3 and remarks afterwards]), with slightly different emphasis. Below, by $\angle(a, b)$, where $a, b \in \mathbb{C} \backslash\{0\}$, we mean the unoriented angle between vectors $a, b$. 
Proposition 4.4. (a) If $\chi \in \mathrm{BMO}$ satisfies $\chi=w+\widetilde{u}+C, C$ a constant, with $\|w\|_{\infty}<\eta<\pi / 2$ and $\|u\|_{\infty}<\Delta<\infty$, then there is $g \in$ $H^{\infty} \cap\left(H^{\infty}\right)^{-1}$ such that

$$
\begin{gathered}
\angle\left(g(\zeta), e^{i \chi(\zeta)}\right)<\eta, \quad \text { a.e. }|d \zeta|, \quad \zeta \in b D, \\
e^{-\Delta} \leq|g(z)| \leq e^{\Delta}, \quad z \in D .
\end{gathered}
$$

Furthermore $\angle\left(g(z), e^{i \chi(z)}\right)<\eta$ for $z \in D$, where $\chi(z)$ is the Poisson integral of $\chi(\zeta), \zeta \in b D$, at $z \in D$.

(b) If $\chi \in \mathrm{VMO}$, then for every $\eta>0$, there are $\Delta<\infty$ and $g \in$ $H^{\infty} \cap\left(H^{\infty}\right)^{-1}$ such that the same conditions (4.4), (4.5) hold.

Proof. (a) Put $g(\zeta):=\exp [u(\zeta)+i v(\zeta)+i C], \zeta \in b D$, where $v=\widetilde{u}$ denotes the conjugate function. Then $g \in H^{\infty}$, and $|g(z)|=\exp u(z) \in$ $\left[e^{-\Delta}, e^{\Delta}\right]$ for $z \in D$. Now

$$
\begin{aligned}
\angle\left(g(\zeta), e^{i \chi(\zeta)}\right) & =\angle\left(g(\zeta) /|g(\zeta)|, e^{i \chi(\zeta)}\right)=\angle\left(e^{i v(\zeta)+i C}, e^{i \chi(\zeta)}\right) \\
& =\angle\left(e^{i[v(\zeta)+C-\chi(\zeta)]}, 1\right)=\angle\left(e^{-i w(\zeta)}, 1\right) \leq|w(\zeta)|<\eta .
\end{aligned}
$$

(b) By [G, Theorem IV.5.2] there are continuous functions $\chi_{1}, \chi_{2}$ such that $\chi=\chi_{1}+\tilde{\chi}_{2}$. Let $\chi_{3}$ be a smooth function such that $\sup _{b D}\left|\chi_{1}-\chi_{3}\right|<\eta$. Then $\tilde{\chi}_{3}$ is a continuous, in particular bounded, function. Set now $w=$ $\chi_{1}-\chi_{3}$ and $u=\chi_{2}-\tilde{\chi}_{3}$. Then $\|w\|_{\infty}<\eta$ and

$$
\|u\|_{\infty} \leq\left\|\chi_{2}\right\|_{\infty}+\left\|\tilde{\chi}_{3}\right\|_{\infty}=: \Delta<\infty .
$$

One can verifiy that $\chi=\left(\chi_{1}-\chi_{3}\right)+\left(\chi_{2}-\tilde{\chi}_{3}\right)^{\sim}+\chi_{3}(0)=w+\widetilde{u}+C$. The conclusion follows from part (a).

Let $C(\zeta, w)$ be a continuous family of cones as in Theorem 4.1 and $\varrho>0$. We define the truncated family of cones $C^{\varrho}$ by the formula

$$
C^{\varrho}(\zeta, w)=C(\zeta, w) \cap \bar{D}(0, \varrho) .
$$

Lemma 4.5. Let $C(\zeta, w)$ be a continuous cone family as in Theorem 4.1. Then there is a positive number $\varrho$ such that for every $\zeta \in b D$, if $K$ is a compact subset of $S_{\zeta}^{\varrho}:=\bigcup_{w \in X_{\zeta}} C^{\varrho}(\zeta, w)$ and is disjoint from $X_{\zeta}$, then its hull $\widehat{K}$ is disjoint from $X_{\zeta}$ as well.

This lemma seems to be intuitively obvious, but the proof that we have is rather long. Since it uses methods extraneous to the rest of the paper, we publish it in a separate note [Sł 5].

Proof of Lemma 4.2. Let $C(\zeta, w), x(\zeta, w), \psi(\zeta, w)$ be as in the Continuous Cone Condition 1.2. For the rest of the proof assume, however, that $C(\zeta, w)$ is already replaced by its truncation $C^{\varrho}(\zeta, w)$, so that the conclusion of Lemma 4.5 holds (i.e. $\beta=\varrho$ ). Fix an arbitrary point $y_{0}=\left(z_{0}, w_{0}\right) \in Y \backslash X$; we are going to show it is in the topological boundary of $\widehat{L}$ in $\mathbb{C}^{2}$. Let 
$\operatorname{gr}(f) \subset Y$ with $f \in H^{\infty}$ be an analytic disc such that $f\left(z_{0}\right)=w_{0}$. By Proposition 2.3, $f \in Q C \cap H^{\infty}$, and by Proposition 4.3(c) the function $\chi(\zeta):=$ $\psi(\zeta, f(\zeta)), \zeta \in b D$, belongs to $Q C$ as well. In particular, $\chi \in$ VMO. Selecting $\eta \in(0, \alpha)$ in Proposition 4.4(b), we obtain a function $g \in H^{\infty} \cap\left(H^{\infty}\right)^{-1}$ and $\Delta<\infty$ such that

$$
\begin{aligned}
e^{-\Delta} \leq|g(z)| \leq e^{\Delta}, & z \in D, \\
\angle(g(\zeta), x(\zeta, f(\zeta)))<\eta, & \text { a.e. }|d \zeta|, \\
\angle\left(g(z), e^{i \chi(z)}\right)<\eta, & z \in D .
\end{aligned}
$$

Let now $g_{t}=f+t g$ for $0<t \leq e^{-\Delta} \beta$. By (4.6) and (4.7),

$$
g_{t}(\zeta) \in C(\zeta, f(\zeta)), \quad \text { a.e. }|d \zeta|, 0<t \leq e^{-\Delta} \beta,
$$

that is, whenever the nontangential limit values $f(\zeta), g(\zeta)$ both exist, but in order to apply Proposition 2.2 we need a more precise relationship, in the sense of cluster values. We recall that $\left(w_{1}, \ldots, w_{n}\right)$ are joint cluster values at $\zeta \in b D$ of functions $f_{1}, \ldots, f_{n} \in H^{\infty}$ if there is a sequence $\left(z_{k}\right)_{k=1}^{\infty} \subset D$ such that $\lim z_{k}=\zeta$ and $\lim _{k} f_{j}\left(z_{k}\right)=w_{j}, j=1, \ldots, n$. We then write $\left(w_{1}, \ldots, w_{n}\right) \in \mathrm{Cl}\left(f_{1}, \ldots, f_{n} ; \zeta\right)$.

Assertion. For every $\left(w_{1}, w_{2}\right) \in \mathrm{Cl}\left(f, g_{t} ; \zeta\right), \zeta \in b D$, and for $t \in$ $\left(0, \beta e^{-\Delta}\right)$,

$$
w_{2} \in C\left(\zeta, w_{1}\right), \quad\left|w_{2}-w_{1}\right| \geq t e^{-2 \Delta} .
$$

Before proving the Assertion, we show that it implies the lemma. We claim

$$
\operatorname{hull}\left(\mathrm{Cl}\left(g_{t}, \zeta\right)\right) \cap L_{\zeta}=\emptyset, \quad \zeta \in b D .
$$

Define $K:=\mathrm{Cl}\left(g_{t}, \zeta\right)$. Observe first that

$$
K=\left\{w_{2}:\left(w_{1}, w_{2}\right) \in \mathrm{Cl}\left(f, g_{t} ; \zeta\right), w_{1} \in \mathrm{Cl}(f, \zeta)\right\} \subset S_{\zeta},
$$

where $S_{\zeta}=\bigcup\left\{C\left(\zeta, w_{1}\right): w_{1} \in X_{\zeta}\right\}$, because of the Assertion and the inclusion $\mathrm{Cl}(f, \zeta) \subset X_{\zeta}, \zeta \in b D$. On the other hand, by the same Assertion and the relation $C\left(\zeta, w_{1}\right) \cap L_{\zeta}=w_{1}$ we obtain

$$
K \cap L_{\zeta}=\emptyset \text {. }
$$

By this relation, if $L_{\zeta} \cap \widehat{K} \neq \emptyset$, then (seeing that $L_{\zeta}$ is connected) it must be contained in one of the connected open components, say $V$, of $\widehat{K} \backslash K$. But then $X_{\zeta} \subset L_{\zeta} \subset V \subset \widehat{K}$, contrary to the disjointness of $X_{\zeta}$ and $\widehat{K}$, stated in Lemma 4.5. Applying Proposition 2.2 with $X^{1}:=L$ and $X_{\zeta}^{2}:=\operatorname{hull}\left(\mathrm{Cl}\left(g_{t}, \zeta\right)\right), \zeta \in b D$, we conclude that $\overline{\operatorname{gr}\left(g_{t}\right)}$ is disjoint from $\widehat{L}$. On the other hand $\lim _{t \rightarrow 0}\left(z_{0}, g_{t}\left(z_{0}\right)\right)=\left(z_{0}, f\left(z_{0}\right)\right)=\left(z_{0}, w_{0}\right)$ (fixed at the beginning of the proof). Thus $\left(z_{0}, w_{0}\right)$ is in the boundary of $\widehat{L}$, ie. $Y \subset b \widehat{L}$.

It remains to prove the Assertion. By the obvious properties of joint clusters, if $\left(w_{1}, w_{2}\right) \in \operatorname{Cl}\left(f, g_{t} ; \zeta\right)$, then there is $w$ such that $\left(w_{1}, w, w_{2}\right) \in$ 
$\mathrm{Cl}\left(f, g, g_{t} ; \zeta\right)$, hence $w_{2}=w_{1}+t w$. Fix a continuous extension

$$
\psi_{1}: \bar{D} \times \bar{D}(0, R) \rightarrow \mathbb{R}
$$

of the given function $\psi: X \rightarrow \mathbb{R}$, where $R>\sup |f|$. With $\chi(z), z \in D$, denoting the Poisson integral of $\chi(\zeta)$, we obtain, by Proposition 4.3(e),

$$
\lim _{n \rightarrow \infty}\left[\psi_{1}\left(z_{n}, f\left(z_{n}\right)\right)-\chi\left(z_{n}\right)\right]=0
$$

for every $\left(z_{n}\right)_{n} \subset D$ with $\left|z_{n}\right| \rightarrow 1$. Applying this to $\left(z_{n}\right)$ such that $z_{n} \rightarrow \zeta$, $f\left(z_{n}\right) \rightarrow w_{1}, g\left(z_{n}\right) \rightarrow w, g_{t}\left(z_{n}\right) \rightarrow w_{1}+t w=w_{2}$, we have

$$
\lim _{n \rightarrow \infty} \psi_{1}\left(z_{n}, f\left(z_{n}\right)\right)=\psi\left(\zeta, w_{1}\right), \quad \lim \chi\left(z_{n}\right)=\psi\left(\zeta, w_{1}\right),
$$

and by (4.8), $\angle\left(g\left(z_{n}\right), e^{i \chi\left(z_{n}\right)}\right)<\eta$, hence $\angle\left(w, e^{i \psi\left(\zeta, w_{1}\right)}\right)<\eta$. Since, by (4.6),

$$
\left|w_{2}-w_{1}\right|=t|w|=\lim \left|\operatorname{tg}\left(z_{n}\right)\right| \in\left[t e^{-2 \Delta}, 1\right],
$$

we obtain the Assertion.

5. Examples. In this section we give several examples which put the earlier results in perspective.

The first example shows that the conclusions of Theorem 3.1 cannot be strengthened, that is, the hull of a family of quasicircles $X_{\zeta}$ satisfying the Continuous Cone Condition at the endpoints might have, in general, nonempty interior.

EXAmPle 5.1. There is a compact subset $X$ of $b D \times \mathbb{C}$ satisfying all the assumptions of Theorem 3.1 such that $\widehat{X} \backslash X$ contains two intersecting analytic discs, and so is not a topological hypersurface. (In fact it has nonempty interior by Corollary 2.1.) Furthermore, the $\operatorname{arcs} X_{\zeta}$ are bilipschitz images of an interval (with a uniform constant), in particular, rectifiable.

We will now describe the example, and in the two assertions that follow, prove its properties.

Construction. We obtain our arcs as bilipschitz images of an interval. The maps will be rotations by a varying angle dependent on the distance to the origin.

Assertion 1. $\operatorname{Let} \Phi(w):=w e^{i s(|w|)}$ for $w \in \mathbb{C} \backslash\{0\}$ and $\Phi(0)=0$, where $s:(0, \infty) \rightarrow \mathbb{R}$ is an $L_{\text {loc }}^{\infty}$ function. Then $\Phi: \mathbb{C} \rightarrow \mathbb{C}$ is quasiconformal if and only if it is bilipschitz, which holds if and only if $\sup _{r \in(0, \infty)} r s^{\prime}(r)<\infty$.

This follows immediately from the computation of the derivatives:

$$
\partial_{w} \Phi=e^{i s(|w|)}\left[1+(i / 2)|w| s^{\prime}(|w|)\right], \quad \partial_{\bar{w}} \Phi=e^{i s(|w|)}(i / 2) s^{\prime}(|w|) w^{2}|w|^{-1} .
$$

Consider now any function $g$, holomorphic on $\bar{D}$, with $g(0)=0$ and such that $\zeta=1$ is the only zero of $g$ on the circumference $b D$; for example $g(z):=$ $z(z-1)$. (We choose these assumptions for the sake of simplicity; our example 
can be substantially generalized.) Let $A(\zeta)$ denote the continuous branch of argument of $g(\zeta)$ on $b D \backslash\{1\}$ with $A(\zeta) \in(0, \infty)$ and $\lim _{\zeta \rightarrow 1} A(\zeta)=0$. For our choice of $g, A$ is bounded, say by $C$. Let now $\Phi_{\zeta}(w):=w e^{i s(\zeta,|w|)}$ for $w \neq 0$ and $\Phi_{\zeta}(0):=0$, where $s(\zeta, r)$ is defined, for a given $\zeta \in b D$, as a piecewise linear function on $[0, \infty)$, interpolating the values $s(\zeta, 0)=0$, $s(\zeta,|g(\zeta)|)=A(\zeta), s(\zeta, 2|g(\zeta)|)=0, s(\zeta, r)=0$ for $r \geq 2|g(\zeta)|$.

Assertion 2. The maps $\Phi_{\zeta}: \mathbb{C} \rightarrow \mathbb{C}, \zeta \in b D$, form a continuous family of uniformly bilipschitz maps such that $\Phi_{\zeta}(|g(\zeta)|)=g(\zeta)$ on $b D$.

The last equation is the essential feature of the construction; the uniform bilipschitz property holds because $r\left|s^{\prime}(\zeta, r)\right| \leq r A(\zeta)|g(\zeta)|^{-1} \leq 2 C$ on $[0,2|g(\zeta)|]$, and is 0 otherwise.

We can now define $X$. We choose a number $b>\sup _{b D} 2|g(\zeta)|$ and let $a=-b$. Set $X_{z}:=\Phi_{\zeta}([a, b])$. We obtain a continuous family of simple arcs joining the constant analytic discs $a$ and $b$ such that $0, g(\zeta) \in X_{\zeta}$ for $\zeta \in b D$. Thus the constant analytic disc 0 and the graph of $g$, which are contained in the hull of $X$, intersect at $(0,0)$, and so the hull has nonempty interior. Observe that the Continuous Cone Condition holds at the endpoints $a, b$, because all the arcs are contained in the strip $\{a \leq \Re w \leq b\}$. All the remaining assumptions of Theorem 3.1 are satisfied. We omit further details.

In the situation of Theorem 3.1 the fact that the given end-discs $a, b$ were shown to be contained in the boundary of this hull, played an essential role in the proof of its properties. We will now show that without assuming the Continuous Cone Condition at the "end" disc $a$, the disc might be contained in the interior of the hull.

ExAMPLE 5.2. There is a compact subset $X$ of $b D \times \mathbb{C}$ satisfying all the asumptions of Theorem 3.1 except for the Continuous Cone Condition at one end-disc, say $a$, such that the disc $a$ is disjoint from $(b Y) \backslash X$. Consequently, $b Y$ cannot be the union of two topological hypersurfaces having the disc $a$ as common part of their boundaries.

The construction is an easy modification of the last one. Retaining the meaning of the symbols $\Phi_{\zeta}: \mathbb{C} \rightarrow \mathbb{C}, \zeta \in b D, g(\zeta)$, and $b$ (together with the underlying choices of $A$ and $s(\cdot))$, we now set $X_{z}:=\Phi_{\zeta}([0, b])$. Here one end-disc $b$ still satisfies the end-disc cone condition while the other, the constant disc $a:=0$, does not, as it intersects over $D$ with the disc $g$. By Corollary 2.1 both discs are contained in $X \cup \operatorname{Int} Y$, and so the Continuous Cone Condition fails along $a=0$.

It follows from Corollary 2.1 that if the hull $Y$ of a family of $\operatorname{arcs} X$ is a topological hypersurface, then no two analytic discs in $Y \backslash X$ can intersect (over $D$ ). The next example shows that, in contrast, the boundaries of two 
closed analytic discs contained in a hypersurface might intersect (over $b D$ ), even if they are continuous at $b D$, and even if the assumptions of Theorem 4.1 are satisfied. We denote by $\tau(\zeta, w)$ the unit tangent vector to $X_{\zeta}$ at $w$, oriented from $a$ to $b$.

EXAMPLE 5.3. There is a family of $\operatorname{arcs}\left(X_{\zeta}\right)$ satisfying the assumptions of Basic Situation 1.1, with all arcs uniformly of class $C^{1}$, and with $\tau(\zeta, w)$ continuous on $X$, such that $Y=\widehat{X}$ is a topological hypersurface and contains two continuous analytic discs intersecting over the boundary $b D$.

Construction. Choose a continuous function $\phi:[-1,0] \rightarrow[0, \infty)$ such that

$$
\begin{gathered}
\phi(-1)=0=\phi(0), \quad \phi(x)>0, \quad-1<x<0, \\
\lim _{x \rightarrow 0} x^{-1} \phi(x)=0,
\end{gathered}
$$

and define a domain $\Omega$ by $\Omega:=\{z=x+i y \in \mathbb{C}:|y|<\phi(x)\}$. Then $\Omega$ is a Jordan domain.

Let $f: \bar{D} \rightarrow \bar{\Omega}$ be the homeomorphic extension of the inverse Riemann mapping function $D \rightarrow \Omega$, partially normalized by the condition $f(-1)=$ $-1, f(1)=0$. Define $x(\zeta)=\Re f(\zeta)$ and $y(\zeta)=\Im f(\zeta)$. It follows from (5.1) and (5.2) that

$$
\begin{gathered}
x(1)=0, \quad-1 \leq x(\zeta)<0, \quad \zeta \in b D \backslash\{1\}, \\
\quad \lim _{\zeta \rightarrow 1} y(\zeta)(\zeta)=0 .
\end{gathered}
$$

Fix $a \geq 2, b \geq 1$. We will construct $X_{\zeta}$ 's by Lagrange interpolation, requiring that $X_{\zeta}$ 's are graphs of cubics $y=y(x)$, over $-a \leq x \leq b$, passing through the four points

$$
-a, x(\zeta)+i y(\zeta), 0, b
$$

if $\zeta \neq 1$, and setting $X_{1}=[-a, b]$. This leads to the parametrization

$$
\gamma(\zeta, t)=t+i \chi(\zeta)(t+a) t(t-b), \quad t \in[-a, b],
$$

where $\chi(1)=0$, and

$$
\chi(\zeta)=(y)(\zeta)[(a+x(\zeta))(x(\zeta)-b)]^{-1}, \quad \zeta \neq 1 .
$$

Using (5.3), (5.4) and the assumptions on $a, b$, we infer that $\chi$ is a continuous function on $b D$ with $\chi(1)=0$. Clearly $\gamma: b D \times[-a, b] \rightarrow \mathbb{C}$ is a continuous function, and for every $\zeta \in b D$,

$$
t \mapsto \gamma(\zeta, t):[-a, b] \rightarrow \mathbb{C}
$$

is a $C^{\omega}$ homeomorphism onto a real-analytic arc, which we define to be $X_{\zeta}$. One can check that $\gamma(x(\zeta))=y(\zeta)$ and $\gamma(0)=0$; hence $f$ and $g=0$ are two analytic discs in $Y=\widehat{X}$ which intersect at $(1,0)$. 
Observe that $\partial_{t} \gamma(\zeta, t)$ is continuous on $b D \times[-a, b]$, because it equals $1+i \chi(\zeta)\left(3 t^{2}+2 a t-2 t b-a b\right)$. Since $|\partial \gamma / \partial t| \geq 1$, the unit tangent vector field $\tau(\zeta, w)$ is continuous on $X$. Hence, the assumptions of Theorem 4.1 are satisfied and so $Y$ is a topological hypersurface.

\section{References}

[F] F. Forstneric, Polynomial hulls of sets fibered over the circle, Indiana Univ. Math. J. 37 (1988), 869-889.

[G] J. B. Garnett, Bounded Analytic Functions, Academic Press, San Diego, 1981.

[MSS] R. Mañé, P. Sad and D. Sullivan, On the dynamics of rational maps, Ann. Sci. École Norm. Sup. 16 (1983), 193-217.

[Sł 1] Z. Słodkowski, Polynomial hulls in $\mathbb{C}^{2}$ and quasicircles, Ann. Scuola Norm. Sup. Pisa Cl. Sci. 16 (1989), 367-391.

[Sł 2] -, Holomorphic motions and polynomial hulls, Proc. Amer. Math. Soc. 111 (1991), 347-355.

[Sł 3] - Extensions of holomorphic motions, Ann. Scuola Norm. Sup. Pisa Cl. Sci. 22 (1995), 185-210.

[Sł 4] - Orientation reversing diffeomorphisms and holomorphic motions, preprint.

[Sł 5] - A generalization of Janiszewski's theorem and an application, preprint.

Department of Mathematics (M/C 249)

University of Illinois at Chicago

851 South Morgan Street

Chicago, IL 60607-7045, U.S.A.

E-mail: zbigniew@uic.edu

Received December 14, 1999

Revised version March 22, 2004 